Pacific Journal of Mathematics

THE SET OF CONTINUOUS NOWHERE DIFFERENTIABL 


\title{
THE SET OF CONTINUOUS NOWHERE DIFFERENTIABLE FUNCTIONS
}

\author{
R. DANiel MaUldin
}

Let $M$ be the set of all continuous real-valued functions defined on the interval $[0,1]$ which do not have a finite derivative anywhere. It is shown that $M$ forms a coanalytic, non-Borel, subset in the space of all real-valued continuous functions on $[0,1]$ provided with the uniform norm.

Let $C$ be the space of all real-valued continuous functions defined on the unit interval provided with the uniform norm. In the Scottish Book, Banach raised the question of the descriptive class of the subset $D$ of $C$ consisting of all functions which are differentiable at each point of $[0,1]$. Banach pointed out that $D$ forms a coanalytic subset of $C$ and asked whether $D$ is a Borel set. Later Mazurkiewicz showed that $D$ is not a Borel set [3].

In this paper, we shall investigate the subset $M$ of $C$ consisting of all functions which do not have a finite derivative at any point of $[0,1]$. It is well known that $M$ is residual in $C$ [2]. We shall prove the following theorem.

THEOREM A. Let $M=\{f \in C: f$ does not have a finite derivative at any point of $[0,1]\}$. The set $M$ is a coanalytic subset of $C$ which is not a Borel set.

In order to see that $C-M$ is an analytic set, notice that a continuous function $f$ has a finite derivative at some point $x$ of $[0,1]$ if and only if for each positive integer $n$, there is a positive integer $m$ so that $\left(^{*}\right)$ if $0<\left|h_{1}\right|,\left|h_{2}\right|<1 / m$ and $x+h_{1}$ and $x+h_{2}$ are both in $[0,1]$, then

$$
\left|\frac{f\left(x+h_{1}\right)-f(x)}{h_{1}}-\frac{f\left(x+h_{2}\right)-f(x)}{h_{2}}\right| \leqq \frac{1}{n} .
$$

For each pair of positive integers $(n, m)$, let $E(n, m)=\{(f, x) \in$ $C \times[0,1]:\left(^{*}\right)$ holds $\}$. Then $C-M$ is the projection into $C$ of $\bigcap_{n=1}^{\infty} \bigcup_{m=1}^{\infty} E(n, m)$. It may be checked that each set $E(n, m)$ is a closed subset of $C \times[0,1]$. Thus, $M$ is a coanalytic subset of $C$. The remainder of this paper is devoted to demonstrating that $M$ is not a Borel set.

Let us make the following conventions. The set of positive integers will be denoted by $N$; by $N^{*}$ shall be meant the set of all finite sequences of positive integers. We shall denote elements of 
$J=N^{N}$ by Greek letters and the terms of such a sequence by its nearest Roman equivalent. Also, if $\sigma=\left\langle s_{k}\right\rangle_{k=1}^{\infty} \in J$ and $n \in N$, then $\sigma \mid n=\left\langle s_{1}, \cdots, s_{n}\right\rangle$.

For each element $s=\left\langle s_{1}, \cdots, s_{k}\right\rangle$ of $N^{*}$, let $I(s)$ be the left open, right closed interval with left end point

$$
a(s)=2^{-s_{1}}+2^{-\left(s_{1}+s_{2}\right)}+\cdots+2^{-\left(s_{1}+\cdots+s_{k}\right)}
$$

and with right end point

$$
b(s)=a(s)+2^{-\left(s_{1}+\cdots s_{k}\right)} .
$$

Notice, that $(0,1]=\bigcup_{p=1}^{\infty} I(\langle p\rangle), I\left(\left\langle s_{1}, \cdots, s_{k}\right\rangle\right)=\bigcup_{p=1}^{\infty} I\left(\left\langle s_{1}, \cdots, s_{k}, p\right\rangle\right)$ and if $s$ and $t$ are distinct elements of $N^{*}$ having the same length, then $I(s)$ and $I(t)$ are disjoint. For each $\sigma \in J$, let $x(\sigma)$ be the point of intersection of $\bigcap_{k=1}^{\infty} I(\sigma \mid k)$. We have $x(\sigma)=\sum_{i=1}^{\infty} 2^{-\left(s_{1}+\cdots+s_{i}\right)}$. For each interval $(a, b]$, set

$$
\varphi_{(a, b]}(x)=\left\{\begin{array}{rll}
x-a, & \text { if } & a<x \leqq(a+b) / 2, \\
b-x, & \text { if } & (a+b) / 2 \leqq x \leqq b, \\
0, & & \text { otherwise } .
\end{array}\right.
$$

For each positive integer $n$, let $h_{n}=\Sigma \varphi_{I(s)}$, where the summation is taken over all elements of $N^{*}$ which have length $n$. Also, let us set $h_{0}(x)=1 / 2-|x-1 / 2|$, for $x \in[0,1]$. For each $n, h_{n}$ is a "sawtooth" function on $[0,1]$. First we give three lemmas concerning these functions.

LeMma 1. For each $n, h_{n}$ is nonnegative and $h_{n}(x) \leqq x /\left(2^{n+1}-1\right)$, for each $x$ in $[0,1]$.

Proof. It can be checked that the line through $(0,0)$ and the highest point of the graph of $h_{n}$ over the interval $I\left(\left\langle s_{1}, \cdots, s_{n}\right\rangle\right)$ has slope $1 / 1+2\left(1+\sum_{i=1}^{n} 2^{s_{i}+\cdots+s_{n}}\right) \leqq 1 /\left(2^{n+1}-1\right)$. This means $h_{n}(x) \leqq$ $x /\left(2^{n+1}-1\right)$, for $x \in[0,1]$.

We will also require the fact that the action of the functions $h_{p}$ is being reproduced on each of the intervals $I\left(\left\langle q_{1}, \cdots, q_{n}\right\rangle\right)$. This is the content of the next lemma which may be proven by induction.

Lemma 2. Let $\left\langle q_{1}, \cdots, q_{n}\right\rangle \in N^{*}$ and let

$$
g(x)=2^{q_{1}+\cdots+q_{n}} x-\left(\frac{1}{2^{q_{1}}}+\cdots+\frac{1}{2^{q_{1}+\cdots+q_{n}}}\right) .
$$

Then $g$ maps $I\left(\left\langle q_{1}, \cdots, q_{n}\right\rangle\right)$ onto $(0,1]$ and for each $p \geqq 0, h_{p}(g(x))=$ $\left(2^{q_{1}+\cdots+q_{n}}\right) h_{n+p}(x)$, for $x \in I\left(\left\langle q_{1}, \cdots, q_{n}\right\rangle\right)$. 
Lemma 3. Let $\left\langle q_{1}, \cdots, q_{2^{k}}\right\rangle \in N^{*}$, then

$$
h_{2^{k+1}}(x) \leqq \frac{1}{2^{2^{k}}}\left(x-\left(\frac{1}{2^{q_{1}}}+\cdots+\frac{1}{2^{q_{1}+\cdots+q_{2^{k}}}}\right)\right),
$$

for each $x \in I\left(\left\langle q_{1}, \cdots, q_{2^{k}}\right\rangle\right)$.

Proof. By Lemma 2, for $x \in I\left(\left\langle q_{1}, \cdots, q_{2^{k}}\right\rangle\right)$

$$
h_{2^{k+1}}(x)=\left(\frac{1}{2^{q_{1}+\cdots+q_{2^{k}}}}\right) h_{2^{k}}(g(x)),
$$

where $g$ is the appropriate function defined in Lemma 2. According to Lemma 1 ,

$$
h_{2^{k+1}}(x) \leqq\left(\frac{1}{2^{q_{1}+\cdots+q_{2} k}}\right) \frac{g(x)}{2^{2^{k+1}}-1} .
$$

Substituting for $g(x)$ and noting that $2^{2^{k}}<2^{2^{k+1}}-1$ :

$$
h_{2^{k+1}}(x) \leqq \frac{1}{2^{2^{k}}}\left(x-\left(\frac{1}{2^{q_{1}}}+\cdots+\frac{1}{2^{q_{1}+\cdots+q_{2} k}}\right)\right) .
$$

It can be shown that $\Sigma h_{n}$ does not have a finite derivative at any $x$ in the $(0,1]$, although we shall not use this fact. However, Theorem A will be demonstrated by continuously modifying a subsequence of $\left\{h_{n}\right\}_{n=1}^{\infty}$. We proceed as follows.

Let $E$ be an analytic subset of the Cantor set $K$. Let $H$ be a map from $N^{*}$ into the clopen subsets of $K$ so that

$$
E=\bigcup_{\sigma \in J} \bigcap_{k=1}^{\infty} H(\sigma \mid k) .
$$

We may assume that $H(\sigma \mid k) \supseteqq H(\sigma \mid n)$ if $n>k$ and $\operatorname{diam}(H(\sigma \mid k))<$ $1 / k[2]$.

For each $q=\left\langle q_{1}, q_{2}, \cdots, q_{2^{i}}\right\rangle \in N^{2^{i}}$, set

$$
\lambda_{q}=1-\chi_{A(q) \cup H\left\langle\left\langle q_{1}, \cdots, q_{2} i-1\right\rangle\right)}
$$

where $A(q)=\bigcup\left\{H(s): s \in N^{2^{i}}\right.$ and $\left.|a(s)-b(q)|<2^{i} /\left(2^{2^{i}+1}-1+2^{i}\right)\right\}$. Of course, $\chi_{B}$ denotes the characteristic function of $B$ on the Cantor set $K$.

For each $n \in N$, set

$$
f_{n}(x, t)=\Sigma \lambda_{s}(t) \varphi_{I(s)}(\chi),
$$

where summation is taken over all elements $s$ of $N^{*}$ of length $2^{n}$.

Let $G(x, t)=\sum_{n=1}^{\infty} f_{n}(x, t)$ and $F(x, t)=t+\sqrt{x}+G(x, t)$, for $(x, t) \in[0,1] \times K$. Finally, define the map $\Gamma$ from $K$ into $C$ by 
setting $\Gamma(t)=F(\cdot, t)$, for each $t$ in $K$. We next note three elementary properties of $\Gamma$.

First, notice that since $f_{n}(x, t) \leqq h_{2^{n}}(x)<2^{-n}$, for each $n$, the series $\Sigma f_{n}(x, t)$ converges uniformly over $[0,1] \times K$. Since, for each $t$, the functions $f_{n}(\cdot, t)$ are continuous, the function $\Gamma(t)$ is an element of $C$. Since $F(0, t)=t, \Gamma$ is one-to-one.

Second, notice that $\Gamma(t)$ does not have a finite derivative at 0 . This is because $(\sqrt{x})^{\prime}(0)=+\infty$ and $G(x, t)-G(0, t) \geqq 0$.

Third, notice that $\Gamma$ is a Borel measurable map of $K$ into $C$. This may be seen by as follows. Define $\Gamma_{n}: K \rightarrow C$ by

$$
\left(\Gamma_{n}(t)\right)(x)=t+\sqrt{x}+\sum_{p=1}^{n} f_{p}(x, t) .
$$

Then $\left\{\Gamma_{n}\right\}_{n=1}^{\infty}$ converges uniformly to $\Gamma$. Also, note that if $(X, M)$ is a measurable space, $Y$ is a metric space and $\left\{f_{n}\right\}_{n=1}^{\infty}$ is a sequence of measurable maps from $X$ into $Y$ and this sequence converges uniformly to $f$, then $f$ is a measurable map. This last fact may be used to verify that each function $\Gamma_{n}$ is Borel measurable and then applied once again to show that $\Gamma$ is Borel measurable.

We shall require some deeper properties of the function $\Gamma$.

Lemma 4. Suppose $\sigma \in J$ and $\{t\}=\bigcap_{n=1}^{\infty} H(\sigma \mid n)$ and $x_{0}=x(\sigma)$. Then $\Gamma(t)$ has a left derivative at $x_{0}$ and $G(\cdot, t)$ has left derivative zero at $x_{0}$.

Proof. It suffices to show that $G(\cdot, t)$ has left derivative zero at $x_{0}$.

Let $\varepsilon>0$. Let $n$ be a positive integer so that $2^{-n}<\varepsilon$. Let $\delta$ be a positive number so that $\left(x_{0}-\delta, x_{0}\right] \subseteq I\left(\sigma \mid 2^{n}\right)$. Since $f_{i}\left(x_{0}, t\right)=0$, for all $i$,

$$
\left|\frac{G(x, t)-G\left(x_{0}, t\right)}{x-x_{0}}\right| \leqq \sum_{i=1}^{n}\left|\frac{f_{i}(x, t)}{x-x_{0}}\right|+\sum_{p=1}^{\infty}\left|\frac{f_{n+p}(x, t)}{x-x_{0}}\right| .
$$

Let $x_{0}-\delta<x<x_{0}$. If $1 \leqq i \leqq n$, then $f_{k}(x, t)=0$. Suppose $p \geqq 1$. Set $\alpha=2^{2^{n+p}+1}-1, \beta=2^{n+p}$ and $d=(\alpha / \alpha+\beta) x_{0}$. If $x \leqq d$, then

$$
\left|\frac{f_{n+p}(x, t)}{x-x_{0}}\right| \leqq \frac{h_{2^{n+p}}(x)}{x_{0}-x} .
$$

Using Lemma 1 and the fact that $1 /\left(x_{0}-x\right) \leqq 1 /\left(x_{0}-d\right)$, we have

$$
\left|\frac{f_{n+p}(x, t)}{x-x_{0}}\right| \leqq \frac{d}{\alpha} \cdot \frac{1}{x_{0}-d}=2^{-(n+p)} .
$$

If $d<x<x_{0}$, then there is some $z=\left\langle z_{1}, \cdots, z_{2^{n+p}}\right\rangle$ so that 


$$
f_{n+p}(x, t)=\lambda_{z}(t) \varphi_{I(z)}(x) \text {. }
$$

If $z=\sigma \mid 2^{n+p}$ then $f_{n+p}(x, t)=0$. Otherwise, $d<b(z) \leqq a\left(\sigma \mid 2^{n+p}\right)<x_{0}$. Thus, $\left|a\left(\sigma \mid 2^{n+p}\right)-b(z)\right|<x_{0}-d=x_{0}(1-\alpha /(\alpha+\beta)) \leqq \beta /(\alpha+\beta)$. This implies that $t$ is in $A(z)$ and therefore $f_{n+p}(x, t)=0$. These considerations lead to the conclusion that $G(\cdot, t)$ has left derivative zero at $x_{0}$.

Let us make the following conventions. The set of all elements of $J$ which are equal to one from some term on will be denoted by $Q$. Let $R(Q)$ denote the set of all $x$ in $[0,1]$ such that there is some element $\sigma \in Q$ for which $x=x(\sigma)$. Notice that $Q$ and $R(Q)$ are countable sets and $\sigma \in J-Q$ if and only if $x(\sigma)$ is in the interior of $I(\sigma \mid k)$, for each $k$.

Lemma 5. Suppose $\sigma \in J-Q, \quad\{t\}=\cap H(\sigma \mid k)$, and $x_{0}=x(\sigma)$. Then $\Gamma(t)$ is differentiable at $x_{0}$.

Proof. In view of Lemma 4 , it suffices to show that $G(\cdot, t)$ has right derivative zero at $x_{0}$.

Let $\varepsilon>0$. Let $n$ be a positive integer so that $2^{-n}<\varepsilon$. Since $\sigma \in J-Q, x_{0}$ is in the interior of $I\left(\sigma \mid 2^{n-1}\right)$. Let $\delta$ be a positive number so that $\left[x_{0}, x_{0}+\delta\right) \subseteq I\left(\sigma \mid 2^{n-1}\right)$ and let $x$ be between $x_{0}$ and $x_{0}+\delta$. Since $f_{k}\left(x_{0}, t\right)=0$, for all $k$, we have

$$
\left|\frac{G(x, t)-G\left(x_{0}, t\right)}{x-x_{0}}\right| \leqq \sum_{i=1}^{n}\left|\frac{f_{i}(x, t)}{x-x_{0}}\right|+\sum_{p=1}^{\infty}\left|\frac{f_{n+p}(x, t)}{x-x_{0}}\right| .
$$

It can be checked that if $1 \leqq i \leqq n$, then $f_{i}(x, t)=0$.

Suppose $p \geqq 1$. If $x \in I\left(\sigma \mid 2^{n+p-1}\right)$, then $f_{n+p}(x, t)=0$. Suppose $b\left(\sigma \mid 2^{n+p-1}\right)<x<x_{0}+\delta$. There is some $q=\left\langle q_{1}, \cdots, q_{2^{n+p-1}}\right\rangle$ so that $x \in I(q)$. Using Lemma 3 , we have

$$
\left|\frac{f_{n+p}(x, t)}{x-x_{0}}\right| \leqq \frac{h_{2^{n+p}}(x)}{x-x_{0}}=\frac{h_{2^{n+p}}(x)}{x-a(q)} \cdot \frac{x-a(q)}{x-x_{0}}<2^{-2^{(n+p-1)}}<2^{-(n+p)} .
$$

It follows from these considerations that $G(\cdot, t)$ has right derivative zero at $x_{0}$.

LEмма 6. If $t$ is in $K-E$, then $\Gamma(t)$ does not have a finite derivative at any point of $[0,1]-R(Q)$.

Proof. We have already noted that $\Gamma(t)$ does not have a finite derivative at 0 . Thus, it suffices to show that $G(\cdot, t)$ does not have a finite derivative at any point of $(0,1)-R(Q)$.

Let $\sigma$ be an element of $J-Q$ and let $x_{0}=x(\sigma)$. 
Suppose there is a positive integer $p_{0}$ so that if $p \geqq p_{0}$, then $\lambda_{o \mid 2^{p}}(t)=0$. If $p \geqq p_{0}$, then $t$ is in $A\left(\sigma \mid 2^{p}\right)$ or $t$ is in $H\left(\sigma \mid 2^{p-1}\right)$. If $t$ were in $H\left(\sigma \mid 2^{p-1}\right)$, for infinitely many $p$, then $t$ would be in $E$. Thus, we may assume that if $p \geqq p_{0}$, then $t$ is in $A\left(\sigma \mid 2^{p}\right)$. For each $p \geqq p_{0}$, there is a point $q^{p}=\left\langle q_{1}^{p}, \cdots, q_{2^{p}}^{p}\right\rangle$ in $N^{2^{p}}$ so that $t$ is in $H\left(q^{p}\right)$ and $\left|a\left(q^{p}\right)-b\left(\sigma \mid 2^{p}\right)\right|<2^{p} /\left(2^{2^{p}+1}-1+2^{p}\right)$. This implies that the sequence $\left\{a\left(q^{p}\right)\right\}_{p=1}^{\infty}$ converges to $x_{0}$. Since $x_{0}$ is in the interior of $I(\sigma \mid 1)$, this implies that there is a positive integer $n_{1}$ so that if $p>n_{1}$, then $q_{1}^{p}=s_{1}$. This means that $t$ is in $H(\sigma \mid 1)$. Similar considerations show that for each $i, t$ is in $H(\sigma \mid i)$. This implies that $t$ is in $E$. This contradicts the assumption that $t$ is not in $E$. Thus, there are infinitely many $p$ such that $\lambda_{o 12^{p}}(t)=1$.

Let $\delta>0$. Choose $p$ so that $\lambda_{\sigma \mid 2 p}(t)=1$ and $(a, b]=I\left(\sigma \mid 2^{p}\right)$ is a subset of $\left(x_{0}-\delta / 2, x_{0}+\delta / 2\right)$. Let $m=(a+b) / 2$. Since $m \neq x_{0}$ and

$$
\begin{aligned}
& \left|\frac{G(b, t)-G(a, t)}{b-a}-\frac{G(b, t)-G(m, t)}{b-m}\right| \\
& \quad=\left|\frac{G(b, t)-G(a, t)}{b-a}-\frac{G(m, t)-G(a, t)}{m-a}\right| \\
& =\left|\frac{f_{p}(b, t)-f_{p}(a, t)}{b-a}-\frac{f_{p}(b, t)-f_{p}(m, t)}{b-m}\right| \\
& =\left|\frac{f_{p}(b, t)-f_{p}(a, t)}{b-a}-\frac{f_{p}(m, t)-f_{p}(a, t)}{m-a}\right|=1,
\end{aligned}
$$

it follows that $G(\cdot, t)$ does not have a finite derivative at $x_{0}[4, \mathrm{pp}$. 114-116].

Let us collect the preceding lemmas together.

Theorem B. There is a countable subset $Y$ of $[0,1]$ such that for each analytic subset $E$ of $K$ there is a one-to-one Borel measurable map $\Gamma$ of $K$ into $C$ and a countable subset $S$ of $E$ so that (1) if $t$ is in $E-S$, then $\Gamma(t)$ has a finite derivative at some point of $[0,1]-Y$ and (2) if $t$ is in $K-E$, then $\Gamma(t)$ does not have a finite derivative at any point of $[0,1]-Y$.

A proof of Theorem A can now be given. Let $Y=\left\{y_{n}\right\}_{n=1}$ be a countable subset of $[0,1]$ so that Theorem $\mathrm{B}$ holds. Let $D(Y)=$ $\{f \in C: f$ has a finite derivative at some point of $[0,1]-Y\}$. It can be shown that $D(Y)$ is an analytic subset of $C$ (in fact, if $Y$ is any coanalytic subset of $[0,1]$, then $D(Y)$ is an analytic subset of $C$ ). Now, if $D(Y)$ were a Borel subset of $C$, then by applying Theorem B, every analytic subset of $K$ would be a Borel subset of $K$. This contradiction establishes that $D(Y)$ is not a Borel subset of $C$. If 
$M$ were a Borel subset of $C$, then $D(Y)$ would be a Borel set, since

$$
D(Y)=(C-M)-\bigcup_{n=1}^{\infty} D_{n},
$$

where $D_{n}=\left\{f \in C: f\right.$ has a finite derivative at $\left.y_{n}\right\}$, and each set $D_{n}$ is an $F_{\sigma o}$ subset of $C$. This contradiction establishes Theorem A.

\section{REFERENCES}

1. R. P. Boas, A Primer of Real Functions, Carus Mathematical Monographs, No. 13, Math. Assoc. Amer.; distributed by Johy Wiley and Sons, New York, 1960.

2. K. Kuratowski, Topology, Volume 1, Academic Press, New York, 1962.

3. S. Mazurkiewicz, Über die Menge der differenzierbaren Functionen, Fund. Math., 27 (1936), 244-249.

4. J. C. Oxtoby, Measure and Category, Springer-Verlag, New York, 1970.

Received August 14, 1978. Research partially supported by NSF grant MCS 78-04376.

North Texas State University

DENTON, TX 76203 



\section{PACIFIC JOURNAL OF MATHEMATICS}

EDITORS

DONALD BABBITT (Managing Editor)

University of California

Los Angeles, California 90024

Hugo RossI

University of Utah

Salt Lake City, UT 84112

C. C. MOORE and ANDREW OGG

University of California

Berkeley, CA 94720

\section{J. DUGUNDJI}

Department of Mathematics University of Southern California Los Angeles, California 90007

R. Finn and J. Milgram Stanford University

Stanford, California 94305

\section{ASSOCIATE EDITORS}
E. F. BECKENBACH
B. H. Neumann
F. WOLF
K. YoSHIDA

\section{SUPPORTING INSTITUTIONS}

UNIVERSITY OF BRITISH COLUMBIA

CALIFORNIA INSTITUTE OF TECHNOLOGY

UNIVERSITY OF CALIFORNIA

MONTANA STATE UNIVERSITY

UNIVERSITY OF NEVADA, RENO

NEW MEXICO STATE UNIVERSITY

OREGON STATE UNIVERSITY

UNIVERSITY OF OREGON
UNIVERSITY OF SOUTHERN CALIFORNIA

STANFORD UNIVERSITY

UNIVERSITY OF HAWAII

UNIVERSITY OF TOKYO

UNIVERSITY OF UTAH

WASHINGTON STATE UNIVERSITY

UNIVERSITY OF WASHINGTON 


\section{Pacific Journal of Mathematics}

\section{Vol. 83, No. 1 \\ March, 1979}

Richard Neal Ball, Topological lattice-ordered groups ............... 1

Stephen Berman, On the low-dimensional cohomology of some

infinite-dimensional simple Lie algebras .................. 27

R. P. Boas and Gerald Thomas Cargo, Level sets of derivatives ......... 37

James K. Deveney and John Nelson Mordeson, Splitting and modularly

perfect fields......................................

Robert Hugh Gilman and Ronald Mark Solomon, Finite groups with small

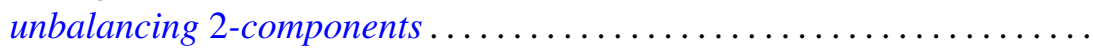

George Grätzer, Andras Hajnal and David C. Kelly, Chain conditions in free products of lattices with infinitary operations..................

Benjamin Rigler Halpern, Periodic points on tori ..................

Dean G. Hoffman and David Anthony Klarner, Sets of integers closed under

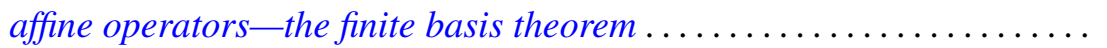

Rudolf-Eberhard Hoffmann, On the sobrification remainder ${ }^{s} X-X \ldots \ldots$

Gerald William Johnson and David Lee Skoug, Scale-invariant

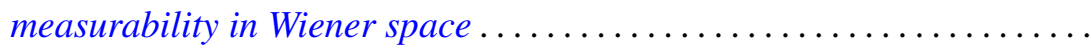

Michael Keisler, Integral representation for elements of the dual of

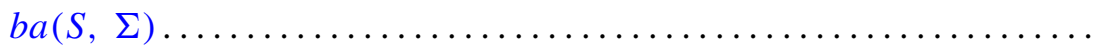

Wayne C. Bell and Michael Keisler, A characterization of the representable Lebesgue decomposition projections ................

Wadi Mahfoud, Comparison theorems for delay differential equations ...

R. Daniel Mauldin, The set of continuous nowhere differentiable functions .

Robert Wilmer Miller and Mark Lawrence Teply, The descending chain condition relative to a torsion theory...

Yoshiomi Nakagami and Colin Eric Sutherland, Takesaki's duality for regular extensions of von Neumann algebras ........ .

William Otis Nowell, Tubular neighborhoods of Hilbert cube manifolds ...

Mohan S. Putcha, Generalization of Lentin's theory of principal solutions of word equations in free semigroups to free product of copies of positive reals under addition

Amitai Regev, A primeness property for central polynomials . ...

Saburou Saitoh, The Rudin kernels on an arbitrary domain. . .

Heinrich Steinlein, Some abstract generalizations of the

Ljusternik-Schnirelmann-Borsuk covering theorem . . . 